Check for updates

Cite this: RSC Adv., 2018, 8, 27045

Received 16th May 2018

Accepted 20th July 2018

DOI: $10.1039 / c 8 r a 04163 f$

rsc.li/rsc-advances

\title{
Surface-initiated atom transfer radical polymerization grafting from nanoporous cellulose gels to create hydrophobic nanocomposites $\uparrow$
}

\author{
Dan Cheng, ${ }^{a}$ Pingdong Wei, ${ }^{a}$ Lina Zhang (D) ${ }^{a}$ and Jie Cai (D) *ab
}

\begin{abstract}
Here, we present the preparation of hydrophobic nanoporous cellulose gel-g-poly(glycidyl methacrylate) (NCG-g-PGMA) nanocomposites by surface-initiated atom transfer radical polymerization (SI-ATRP) of glycidyl methacrylate (GMA) monomers and hydrophobic modification with pentadecafluorooctanoyl chloride $\left(\mathrm{C}_{7} \mathrm{~F}_{15} \mathrm{COCl}\right)$ on the cellulose nanofibrils of the NCG. The successful grafting of PGMA and hydrophobic modification of $\mathrm{C}_{7} \mathrm{~F}_{15} \mathrm{CO}$ - groups on the $\mathrm{NCG}$ was evaluated by Fourier transform infrared (FTIR) spectroscopy. X-ray diffraction (XRD) and scanning electron microscopy (SEM) confirmed that the SI-ATRP and hydrophobic modification did not change the microscopic morphology and structure of the NCG-g-PGMA nanocomposites. Dynamic mechanical analysis (DMA) and thermogravimetric analysis (TGA) showed remarkable thermomechanical properties and moderate thermal stability. The method has tremendous promise to use NCG as a platform for SI-ATRP and produce new functional NCG-based nanomaterials.
\end{abstract}

\section{Introduction}

Nanocellulose, such as cellulose nanocrystals/nanowhiskers (CNC/CNW), nanofibrillated cellulose (NFC) and bacterial cellulose (BC), has attracted special attention because of its remarkable chemical and physical properties, such as high reactivity, higher elastic modulus than that of carbon nanotubes of 100 to $200 \mathrm{GPa}$, and lower thermal expansivity than that of silica of $0.1 \mathrm{ppm} \mathrm{K}^{-1}$ as well as its sustainability, high availability, and low cost..$^{1-3}$ Benefitting from these unique nanostructures and fascinating properties, nanocellulose-based materials are, therefore, of great interest in new technology fields, such as green flexible electronic devices, biological platforms and energy storage materials, especially when the increasing environmental concerns are considered. ${ }^{4-9}$

Because of the natural advantage created by the abundance of hydroxyl groups, the use of nanocellulose in more advanced applications can typically be achieved by performing modifications to alter or tailor the chemical structure and physical properties of nanocellulose, providing access to new application possibilities. Different chemical modification approaches have been attempted, including non-covalent surface modification, sulfonation, 2,2,6,6-tetramethylpiperidine-1-oxyl-mediated oxidation (TEMPO), esterification, etherification, silylation,

${ }^{a}$ College of Chemistry and Molecular Sciences, Wuhan University, Wuhan 430072, China. E-mail: caijie@whu.edu.cn; Tel: +86-27-6878-9321

${ }^{b}$ Research Institute of Shenzhen, Wuhan University, Shenzhen 518057, China

$\dagger$ Electronic supplementary information (ESI) available. See DOI: $10.1039 / \mathrm{c} 8 \mathrm{ra} 04163 \mathrm{f}$ click chemistry, polymer grafting and so on. ${ }^{3}$ Among these surface-modification approaches, surface-initiated atom transfer radical polymerization (SI-ATRP) is an effective method and has been widely used to tailor the chemical and physical properties at interfaces of metals, plastics, inorganic materials, biomolecules and so on. ${ }^{10-14}$ Compared to reversible addition fragmentation transfer (RAFT) or nitroxide mediated polymerization (NRP) reactions, macroinitiators can be easily immobilized on the hydroxyl groups of cellulose so that different polymer structures can be prepared.$^{15}$ This reaction tolerates a wide variety of functional monomer and has also been successfully applied to the grafting well-defined synthetic polymers on the surface of nanocellulose. ${ }^{16}$ Many efforts to prepare polymer-grafted cellulose via SI-ATRP have been reported, and numerous new properties have been achieved. For example, enhanced hydrophobicity was accomplished by grafting various monomers (methyl methacrylate, butyl acrylate, styrene, glycidyl methacrylate and perfluorooctylethyl methacrylate) ${ }^{17-20}$ stimuli-responsive properties were obtained by grafting poly(poly(ethylene glycol)methylacrylate)s, poly( $N$ isopropylacrylamide), poly(dimethylaminoethy methacrylate), poly(diethylaminoethyl methacrylate), poly(4-vinylpyridine); ${ }^{21-24}$ improved biofunctionization (low-adherent wound dressings, DNA hybridization detection, protein purification, and so on) were developed by grafting poly( $N, N$-dimethylaminoethylmethacrylate):;5-27 and reinforced mechanical properties were achieved by preparing polymer nanocomposites. ${ }^{28-31}$

Although the majority of reported studies deal with CNC, CNW, CNF and BC with native crystallinity (cellulose I), nanoporous cellulose gel (NCG) with cellulose II crystallinity 
developed from aqueous alkali hydroxide/urea solutions is composed of interconnected cellulose nanofibrils. ${ }^{32}$ Moreover, the NCG showed remarkable mechanical strength, high porosity, light transmittance, and good formability. ${ }^{33,34}$ Due to its excellent performance and special three-dimensional structure, NCG become an ideal functional platform for the preparation of cellulose-based nanomaterials with specific functions. For example, NCG has been used as a template or support for the synthesis of inorganic nanomaterials ${ }^{35-37}$ and conductive polymers, ${ }^{38,39}$ as a precursor for cellulose bioplastics, ${ }^{40}$ and as a 3D reinforcing phase for polymer nanocomposites. ${ }^{33,41,42}$ However, NCG-based materials are inherently hydrophilic, and most cellulosic materials can swell significantly in water, limiting their application range to a large extent. Hydrophobic modification is a practical method for increasing the application field of cellulose-based materials. Herein, we demonstrate that the interconnected nanofibrillar network structure of the NCG was grafted with PGMA. Further hydrophobic modification with $\mathrm{C}_{7} \mathrm{~F}_{15} \mathrm{COCl}$ did not change the microscopic morphology and structure of the NCG- $g$-PGMA nanocomposites, leading to a remarkable improvement in the hydrophobicity and tensile storage modulus $\left(E^{\prime}\right)$ above the glass transition temperature $\left(T_{\mathrm{g}}\right)$. To the best of our knowledge, this is the first example of NCG as platform for SI-ATRP. This promising synthetic strategy provides potential opportunities for fabricating functional NCG-based nanomaterials.

\section{Experimental section}

\section{Materials}

Cellulose (cotton linter pulp) with its viscosity-average molecular weight $\left(M_{\eta}\right)$ of $9.2 \times 10^{4}$ was supplied by Sanyou Chemical Fibre Co., Ltd. (Tangshan, China) and the material was dried under vacuum at $60{ }^{\circ} \mathrm{C}$ for $24 \mathrm{~h}$ prior to use. $\alpha$-Bromoisobutyryl bromide (BiB), $\mathrm{C}_{7} \mathrm{~F}_{15} \mathrm{COCl}$, glycidyl methacrylate (GMA) and $N, N, N^{\prime}, N^{\prime \prime}, N^{\prime \prime}$ pentamethyldiethylenetriamine (PMDETA) were purchased from Aladdin (Shanghai, China). Triethylamine (TEA), 4-dimethylaminopyridine (DMAP), copper(II) bromide $\left(\mathrm{CuBr}_{2}\right)$, copper(I) chloride $(\mathrm{CuCl})$, tetrahydrofuran (THF), toluene, concentrated hydrochloric acid ( $\mathrm{HCl})$, ethanol, methanol, dichloromethane (DCM), lithium hydroxide hydrate, and urea were purchased from Shanghai Chemical Reagent Co., Ltd., China. GMA was passed through a column of neutral aluminum oxide prior to use to remove the inhibitor. All other chemicals were used as received without any further purification.

\section{Fabrication of nanoporous cellulose gel (NCG)}

Cellulose was dissolved in an aqueous $4.6 \mathrm{wt} \% \mathrm{LiOH} / 15 \mathrm{wt} \%$ urea solution precooled to $-12{ }^{\circ} \mathrm{C}$ to form a $6 \mathrm{wt} \%$ transparent cellulose solution according to our previous method. ${ }^{\mathbf{4 3}}$ After removing air bubbles by centrifugation, the cellulose solution was spread on a glass plate in a $1 \mathrm{~mm}$-thick layer following regeneration by ethanol to give cellulose alcogels. The cellulose alcogels were thoroughly washed with deionized water to achieve NCG hydrogels. The water content of the NCG hydrogel was $90 \%$.

\section{Immobilization of initiator on the NCG}

The procedure for immobilizing the initiator on the NCG surface was performed according to the method of Carlmark and Malmstrom. ${ }^{\mathbf{4 4 , 4 5}}$ Prior to immobilization of the initiator, the NCG hydrogels (0.72 g, $1.33 \mathrm{mmol} \mathrm{OH}$ groups) were converted to NCG-THF organogels by solvent exchange with THF. Then, the available hydroxyl groups on the NCG were converted into SIATRP initiators by immersing the NCG-THF organogels in a solution containing BiB (305 mg, $1.33 \mathrm{mmol})$, TEA (148 mg, $1.46 \mathrm{mmol})$, and a catalytic amount of DMAP in THF $(10 \mathrm{ml})$. The reaction was allowed to proceed overnight at room temperature on a shaking device. Thereafter, the initiatorimmobilized NCG (NCG-BiB) was thoroughly washed in THF to remove residual reactants and byproducts and then subjected to solvent exchange with toluene. The byproduct was the white triethylammonium bromide. ${ }^{46}$

\section{Grafting of GMA on the NCG-BiB by SI-ATRP}

The NCG-BiB obtained from the previous step was placed in a $25 \mathrm{ml}$ round-bottomed flask containing toluene (4 ml), GMA (4.30 g, $30.2 \mathrm{mmol}$ ), $\mathrm{CuBr}_{2}$ (4 mg, $18 \mu \mathrm{mol}$ ), CuCl (14 mg, 141 $\mu \mathrm{mol})$, and PMDETA (25 mg, $140 \mu \mathrm{mol})$. The flask was sealed with a rubber septum and thereafter evacuated and back-filled with argon three times before being placed in a thermostated water bath. Grafting of GMA on the NCG-BiB was conducted between 0 and $30{ }^{\circ} \mathrm{C}$, and the reaction was allowed to proceed for 5 to $48 \mathrm{~h}$. After polymerization, the obtained NCG- $g$-PGMA nanocomposite was thoroughly washed in THF, DCM, THF $/ \mathrm{H}_{2} \mathrm{O}$ and $\mathrm{MeOH}$, successively, to remove residual monomer and the catalyst complex.

To estimate the weight gain, the specimens were weighed before and after polymerization. The weight gain ( $W_{\text {PGMA }}, w \mathrm{t} \%$ ) was calculated as follows:

$$
W_{\mathrm{PGMA}}=\frac{W_{2}-W_{1}}{W_{1}} \times 100 \%
$$

where $W_{1}$ is the estimated dry weight of the NCG-BiB and $W_{2}$ is the dry weight of the NCG- $g$-PGMA nanocomposites.

\section{Hydrophobic modification on hydrolyzed NCG- $g$-PGMA nanocomposites with $\mathrm{C}_{7} \mathrm{~F}_{15} \mathrm{COCl}$}

The epoxide groups of PGMA in the NCG- $g$-PGMA nanocomposites were hydrolyzed with $\mathrm{HCl}\left(0.64 \mathrm{mmol} \mathrm{l}^{-1}\right)$ in a THF$\mathrm{H}_{2} \mathrm{O}$ mixture. The reaction was allowed to proceed for $12 \mathrm{~h}$; the NCG- $g$-PGMA nanocomposites were subsequently rinsed with THF and $\mathrm{CH}_{2} \mathrm{Cl}_{2}$, successively. The hydrolyzed NCG- $g$-PGMA nanocomposite was then transferred to a round-bottomed flask containing DCM $(10 \mathrm{ml})$, TEA $(74.0 \mathrm{mg}, 730 \mu \mathrm{mol})$, and DMAP (catalytic amount). $\mathrm{C}_{7} \mathrm{~F}_{15} \mathrm{OCl}(288 \mathrm{mg}, 670 \mu \mathrm{mol})$ was added dropwise, and the acylation reaction was allowed to proceed overnight. Thereafter, the NCG- $g$-PGMA nanocomposites were thoroughly washed in DCM and ethanol, successively, to remove residual reactants and byproducts. The hydrophobic NCG-g-PGMA nanocomposites were finally dried in a vacuum oven at $60{ }^{\circ} \mathrm{C}$. Reference sample of $\mathrm{NCG}-\mathrm{C}_{7} \mathrm{~F}_{15}$ was prepared using $\mathrm{C}_{7} \mathrm{~F}_{15} \mathrm{OCl}$. Prior to reaction, the NCG hydrogel was 
converted to NCG-DCM gel by solvent exchange with DCM. The acylation reaction was performed in a similar manner using the same molar amounts of $\mathrm{C}_{7} \mathrm{~F}_{15} \mathrm{OCl}$ as those in the example above. Reference sample of neat PGMA was prepared using in situ polymerization. The GMA contained $5 \%(\mathrm{w} / \mathrm{v})$ benzoyl peroxide as initiator was subsequently sealed into glass mold to prevent solution evaporation, and heated at $47^{\circ} \mathrm{C}$ for $12 \mathrm{~h}$ to polymerize and then $90{ }^{\circ} \mathrm{C}$ for $3 \mathrm{~h}$ to remove the residual monomer. ${ }^{33}$ Hydrophobic NCG-g-PGMA nanocomposites were designated as $\mathbf{S 1 - S 4}$ and $\mathrm{S} 3, \mathrm{~S} 5-\mathrm{S} 8$, and the reaction conditions were $0-30{ }^{\circ} \mathrm{C}$ for $24 \mathrm{~h}$ and $5-48 \mathrm{~h}$ at $20{ }^{\circ} \mathrm{C}$, respectively. The reaction condition and properties of nanocomposites is listed in Table 1.

\section{Characterization}

Fourier transform infrared (FTIR) spectra were recorded at ambient temperature on an FTIR spectrometer (Nicolet 5700 FTIR Spectrometer, MA) in the wavelength range from 4000 to $400 \mathrm{~cm}^{-1}$ with a $2 \mathrm{~cm}^{-1}$ resolution and an accumulation of 32 scans.

Wide-angle X-ray diffraction (WAXD) measurements were carried out on a WAXD diffractometer (D8-Advance, Bruker Corporation). The X-rays used were Ni-filtered $\mathrm{CuK}_{\alpha}$ radiation with a wavelength of $1.542 \AA$. The voltage was set at $40 \mathrm{kV}$, and the current was set at $40 \mathrm{~mA}$. The samples were mounted on a solid circular holder, and the proportional counter detector was set to collect data at a rate of $3^{\circ} \mathrm{min}^{-1}$ over the $2 \theta$ range from $5^{\circ}$ to $40^{\circ}$.

Scanning electron microscopy (SEM) observations of the surface of the NCG, NCG- $g$-PGMA nanocomposite and hydrophobic NCG-g-PGMA nanocomposites were performed with a Zeiss Sigma microscope. The imbibed water of the NCG was exchanged with tert-butyl alcohol $(t-\mathrm{BuOH})$, and then the hydrogel was freeze dried to give an aerogel. Before observation, the NCG, NCG-g-PGMA nanocomposite and hydrophobic NCG$g$-PGMA nanocomposites were coated with gold.

${ }^{1} \mathrm{H}$ NMR spectra were recorded on a Bruker Avance III HD $400 \mathrm{MHz}$ NMR instrument, using $\mathrm{CDCl}_{3}$ as solvent. Dibromomethane peak was used as internal standard.

The molecular weight and molecular weight distributions of the cleaved PGMA obtained by hydrolysis of the NCG- $g$-PGMA nanocomposites were measured by gel permeation chromatography (GPC, Waters Instruments, American). The hydrolysis procedure was as follows: $0.04 \mathrm{~g}$ of the NCG- $\mathrm{g}$-PGMA nanocomposite was placed in a round-bottomed flask containing $15 \mathrm{ml}$ of $1.5 \mathrm{~mol} \mathrm{l}^{-1}$ aqueous $\mathrm{HCl}$. The flask was stirred at $90{ }^{\circ} \mathrm{C}$ for $72 \mathrm{~h}$. The reaction mixture was filtered to separate the solid cellulose particles, and the aqueous $\mathrm{HCl}$ was removed by evaporation. The weight average molecular weight $\left(M_{\mathrm{w}}\right)$ and number average molecular weight $\left(M_{\mathrm{n}}\right)$ of the cleaved PGMA were determined by GPC on an instrument equipped with a Waters 717 plus autosampler, a Waters 1515 isocratic HPLC pump, a Waters 2414 refractive index detector, and Shodex K805, K-804, and K-802.5 columns in series. The GPC apparatus was calibrated with polystyrene standards, and THF was used as the eluent $\left(1.0 \mathrm{ml} \mathrm{min}{ }^{-1}\right)$. Measurements were performed at $30{ }^{\circ} \mathrm{C}$ (both the columns and the detector).

The water contact angles (CA) of the NCG, NCG-g-PGMA nanocomposite and hydrophobic NCG-g-PGMA nanocomposites were evaluated on a contact angle system (OCA20, Germany). One drop of water $(2 \mu \mathrm{l})$ was dropped on the sample surface with an automatic piston syringe and photographed.

Differential scanning calorimetry (DSC) was performed using a DSC Q20 differential scanning calorimeter (TA Instruments, USA) to evaluate the glass transition temperature $\left(T_{\mathrm{g}}\right)$ of the PGMA and hydrophobic NCG-g-PGMA nanocomposites. Approximately $10 \mathrm{mg}$ of the sample was placed in a DSC cell. Each sample was heated from 30 to $150{ }^{\circ} \mathrm{C}$ at $10^{\circ} \mathrm{C} \mathrm{min}{ }^{-1}$, kept at $150{ }^{\circ} \mathrm{C}$ for $2 \mathrm{~min}$, and then cooled down at same rate.

Dynamic mechanical analysis (DMA) temperature sweeps under oscillatory stress were performed on a DMA Q800 system (TA Instruments, USA) in tensile mode at a heating rate of $3{ }^{\circ} \mathrm{C} \min ^{-1}$ and with a distance between jaws of $10 \mathrm{~mm}$ in the temperature range from 35 to $180^{\circ} \mathrm{C}$ with a frequency of $1 \mathrm{~Hz}$. The width of the samples was approximately $10 \mathrm{~mm}$.

Thermogravimetric analysis (TGA) analysis was performed on an STA 449C thermal analyzer (NETZSCH, Germany) under nitrogen at a heating rate of $10{ }^{\circ} \mathrm{C} \mathrm{min}-1$ from 30 to $600{ }^{\circ} \mathrm{C}$.

\section{Results and discussion}

Fig. 1 shows the synthetic approach toward the hydrophobic NCG- $g$-PGMA nanocomposites by SI-ATRP of GMA monomer on the surface of the NCG and functionalization of $\mathrm{C}_{7} \mathrm{~F}_{15} \mathrm{COCl}$. The

Table 1 Physical properties of the hydrophobic NCG-g-PGMA nanocomposites ${ }^{a}$

\begin{tabular}{|c|c|c|c|c|c|c|c|c|c|}
\hline Sample & $t, \mathrm{~h}$ & $T,{ }^{\circ} \mathrm{C}$ & $\rho, \mathrm{g} \mathrm{cm}^{-3}$ & Conv., \% & $W_{\text {PGMA }}, \mathrm{wt} \%$ & $M_{\mathrm{n}}, \mathrm{g} \mathrm{mol}^{-1}$ & $M_{\mathrm{w}} / M_{\mathrm{n}}$ & $\mathrm{CA},{ }^{\circ}$ & $T_{\mathrm{g}},{ }^{\circ} \mathrm{C}$ \\
\hline S1 & 24 & 0 & 0.8 & 17 & 63 & 1500 & 1.03 & 75 & 85 \\
\hline S2 & 24 & 10 & 0.9 & 27 & 67 & 7900 & 1.54 & 112 & 83 \\
\hline S4 & 24 & 30 & 1.3 & 54 & 77 & 9000 & 1.53 & 121 & 72 \\
\hline S5 & 5 & 20 & 0.86 & 16 & 63 & 4300 & 1.20 & 79 & 84 \\
\hline S6 & 10 & 20 & 0.96 & 17 & 67 & 6000 & 1.36 & 86 & 83 \\
\hline
\end{tabular}

${ }^{a}$ Monomer conversion (Conv.) calculated from ${ }^{1} \mathrm{H}$ NMR. The $W_{\mathrm{PGMA}}$ was calculated by eqn (1). $M_{\mathrm{n}}$ and $M_{\mathrm{w}}$ were number average molecular weight and weight average molecular weight for PGMA side chains calculated from GPC. CA is the water contact angle. The $T_{\mathrm{g}}$ was determined by DSC. 


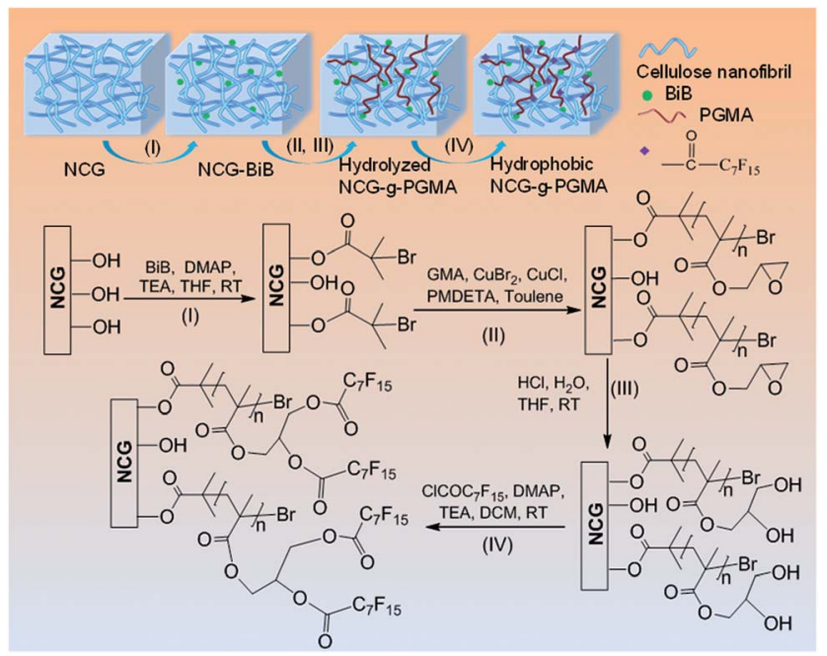

Fig. 1 Synthetic approach toward hydrophobic NCG-g-PGMA nanocomposites by SI-ATRP and hydrophobic modification: (I) immobilization of the initiator $(\mathrm{BiB})$ onto the cellulose nanofibrils of the NCG; (II) grafting of GMA on the NCG-BiB by SI-ATRP; (III) hydrolysis of the NCG-g-PGMA nanocomposite; (IV) hydrophobic modification of $\mathrm{C}_{7} \mathrm{~F}_{15} \mathrm{COCl}$ on the hydrolyzed NCG-g-PGMA nanocomposite.

NCG possesses an interconnected cellulose nanofibrillar network structure, resulting in a large pore volume and specific surface area suitable for in situ polymerization. The abundant hydroxyl groups on cellulose reacts with $\mathrm{BiB}$ so that the initiator is immobilized on the cellulose surface, becoming a SI-ATRP initiator in the graft polymerization of GMA monomer. ${ }^{47}$ The NCG hydrogel was subjected to solvent exchange to form a NCGTHF organogel, which was then immersed in a THF solution containing BiB as an initiator and TEA and DMAP as catalysts at ambient temperature to give the initiator-immobilized NCG (NCG-BiB). After removing residual reactants and byproducts, the NCG-BiB was subjected to solvent exchange with toluene. Subsequently, the NCG-BiB toluene organogel was immersed in a solution of GMA monomer in toluene that also contained $\mathrm{CuBr}_{2}, \mathrm{CuCl}$ and PMDETA as catalysts to obtain an NCG- $g$ PGMA nanocomposite by SI-ATRP. Then, the NCG- $g$-PGMA nanocomposite was thoroughly washed with THF, DCM, THF/ $\mathrm{H}_{2} \mathrm{O}$ and $\mathrm{MeOH}$ to remove residual monomer and catalysts. The epoxide groups of PGMA in the NCG- $g$-PGMA nanocomposite were converted to hydroxyl groups by hydrolysis in a solution of THF, $\mathrm{H}_{2} \mathrm{O}$ and $\mathrm{HCl}$ to give the hydrolyzed NCG- $g$-PGMA nanocomposite. Subsequently, the hydrolyzed NCG- $g$-PGMA nanocomposite was reacted with $\mathrm{C}_{7} \mathrm{~F}_{15} \mathrm{OCl}$ in a DCM solution containing TEA and DMAP as catalysts via acylation. The hydrophobic NCG- $g$-PGMA nanocomposite was then obtained after thoroughly washing the product with DCM and ethanol and subsequent drying.

Fig. 2 shows the FTIR spectra of the NCG, NCG-BiB, NCG- $g$ PGMA nanocomposite and hydrophobic NCG- $g$-PGMA nanocomposite (Table 1, sample S3). The broad and unstructured band in the $\mathrm{OH}$ stretching vibration region (3100 to $3700 \mathrm{~cm}^{-1}$ ) of the cellulose vibrational spectrum contains information about intra- and intermolecular hydrogen bonding interactions.

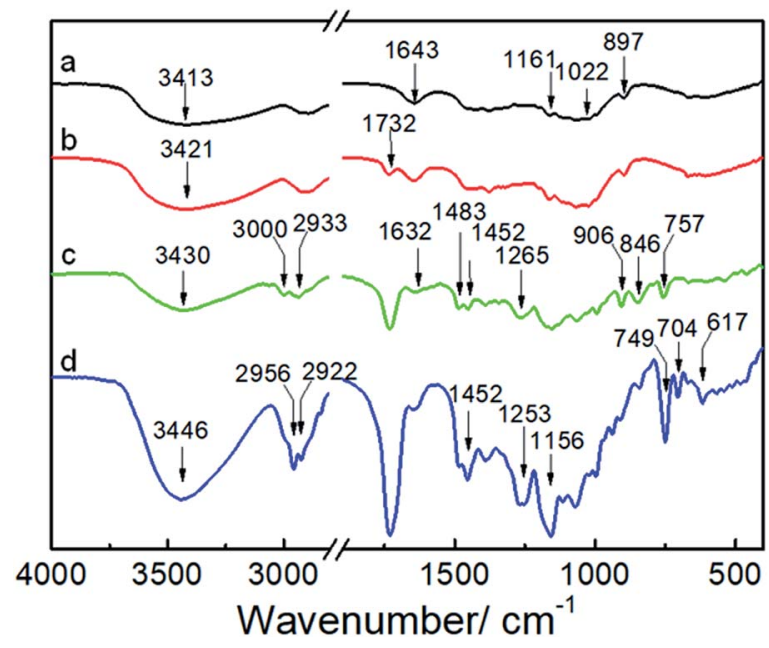

Fig. 2 FTIR spectra of the NCG (a), NCG-BiB (b), NCG-g-PGMA nanocomposite (c) and hydrophobic NCG-g-PGMA nanocomposite (Table 1, sample S3) (d).

The typical FTIR bands of the NCG are visible, including the bands attributed to the stretching vibrations of $\mathrm{O}-\mathrm{H}$ $\left(3413 \mathrm{~cm}^{-1}\right)$, antisymmetric $\mathrm{C}-\mathrm{O}-\mathrm{C}$ glycoside $\left(1161 \mathrm{~cm}^{-1}\right), \mathrm{C}-\mathrm{O}$ in $\mathrm{C}-\mathrm{OH}$ groups $\left(1022 \mathrm{~cm}^{-1}\right)$ and $\mathrm{C}-\mathrm{O}-\mathrm{C}$ bridges $\left(897 \mathrm{~cm}^{-1}\right)$ (Fig. 2a). ${ }^{48}$ The peak observed at $1643 \mathrm{~cm}^{-1}$ was assigned to the $\mathrm{H}-\mathrm{O}-\mathrm{H}$ angle vibration. Compared to the FTIR spectrum of the NCG, the appearance of a new carbonyl ester stretching vibration at $1732 \mathrm{~cm}^{-1}$ in the spectrum of the NCG-BiB indicates the immobilization of $\mathrm{BiB}$ on cellulose (Fig. 2b). ${ }^{24,28}$ Fig. 2c shows the appearance of signals corresponding to the NCG- $g$-PGMA nanocomposites after SI-ATRP. The strong peak observed at $1732 \mathrm{~cm}^{-1}$ is attributed to the ester carbonyl stretching of the GMA units. The other bands seen at $906 \mathrm{~cm}^{-1}$ and $846 \mathrm{~cm}^{-1}$ were due to asymmetric stretching of the epoxy groups. The peak appearing at $757 \mathrm{~cm}^{-1}$ possibly represents the $-\mathrm{CH}_{2}$ rocking vibration of the oxirane groups. Furthermore, successful polymerization was further confirmed by the appearance of four new strong absorption bands at 3000, 2933, 1483 and $1452 \mathrm{~cm}^{-1}$, which are assigned to the $\mathrm{C}-\mathrm{H}$ stretching and bending vibrations of the methylene and methyl groups in the PGMA chains. ${ }^{49,50}$ The band observed at $1265 \mathrm{~cm}^{-1}$ is characteristic of the stretching vibrations of $\mathrm{C}-\mathrm{O}-\mathrm{C}$ in the polymer chains. The shift in this peak from 1643 to $1632 \mathrm{~cm}^{-1}$ and the decrease in its intensity likely results from a decrease in the $\mathrm{H}-\mathrm{O}-\mathrm{H}$ interactions between PGMA and the NCG. Compared with the NCG- $g$-PGMA nanocomposite, the hydrophobic NCG- $g$ PGMA nanocomposite (Table 1, sample S3) exhibited C-O-C stretching vibrations of the polymer chains at 1265 and $1253 \mathrm{~cm}^{-1}$, a C-F symmetrical stretching peak at $1156 \mathrm{~cm}^{-1}$, a C-C skeleton vibration of straight-chain alkanes at $704 \mathrm{~cm}^{-1}$ and a $\mathrm{C}-\mathrm{Br}$ vibration absorption peak at $617 \mathrm{~cm}^{-1}$ (Fig. 2d). ${ }^{51}$ Although functionalization with $\mathrm{C}_{7} \mathrm{~F}_{15} \mathrm{OCl}$ might also occur at the hydroxyl group of NCG, the primary hydroxyl groups at hydrolyzed NCG- $g$-PGMA nanocomposite had higher reactivity because the hydroxyl group of NCG were sterically hindered by PGMA chains. ${ }^{16}$ Moreover, the peak observed at $1732 \mathrm{~cm}^{-1}$ in the spectra of the NCG-BiB, NCG-g-PGMA nanocomposite and 
hydrophobic NCG-g-PGMA nanocomposite indicates that a large amount of ester groups were introduced into the NCG (Fig. 2b-d), confirming again the successful grafting of $\mathrm{BiB}$, GMA and $\mathrm{C}_{7} \mathrm{~F}_{15} \mathrm{COCl}^{52}$ The shift in the $\mathrm{C}-\mathrm{H}$ stretching absorption bands of the methylene and methyl groups in the polymer chains from 3000 and $2933 \mathrm{~cm}^{-1}$, respectively, to 2956 and $2922 \mathrm{~cm}^{-1}$ and the increased intensity of the $-\mathrm{CH}_{2}$ rocking vibration at $749 \mathrm{~cm}^{-1}$ likely result from grafting of the hydrophobic compound $\left(\mathrm{C}_{7} \mathrm{~F}_{15} \mathrm{OCl}\right)$ on the NCG- $g$-PGMA nanocomposite. The FTIR spectra of the NCG, NCG-BiB, NCG- $g$ PGMA nanocomposite and hydrophobic NCG- $g$-PGMA nanocomposite (sample S3) showed the characteristic stretching vibrations of hydroxyl groups in the range from 3300 to $3500 \mathrm{~cm}^{-1}$. However, this peak and intensity of the hydrogen bonding interactions became narrower and weaker with the grafting of PGMA and $\mathrm{C}_{7} \mathrm{~F}_{15} \mathrm{OCl}$, and the hydroxyl peak shifted from 3421 to $3446 \mathrm{~cm}^{-1}$, indicating that hydrogen bonding interactions occurred between the NCG and PGMA.

$\mathrm{X}$-ray diffraction (XRD) was used to examine the aggregate structure of the NCG, PGMA, and hydrophobic NCG- $g$-PGMA nanocomposites (Fig. 3). The XRD pattern of the NCG showed three characteristic peaks centered at $12.0^{\circ}, 20.6^{\circ}$ and $21.8^{\circ}$, corresponding to the typical (110), (110), and (200) crystalline planes of cellulose II, respectively (Fig. 3a). ${ }^{32}$ PGMA displayed a diffraction pattern typical of an amorphous polymer with broad bands centered at approximately $18.8^{\circ}$ and $30.7^{\circ}$ (Fig. $3 \mathrm{~b}$ ). Although the characteristic peak intensity of NCG in hydrophobic NCG- $g$-PGMA nanocomposites decreases at $12.0^{\circ}, 20.6^{\circ}$ and $21.8^{\circ}$, the $(1 \overline{1} 0)$ and (200) crystal planes of cellulose II still existed obviously. The XRD patterns of the hydrophobic NCG- $g$ PGMA nanocomposites (Table 1, sample S1-S4) prepared at different temperatures exhibited the characteristics of PGMA and NCG (Fig. 3c-f). These features demonstrate that the XRD patterns of the hydrophobic NCG- $g$-PGMA nanocomposites (sample S1-S4) nearly become a superposition of those of neat

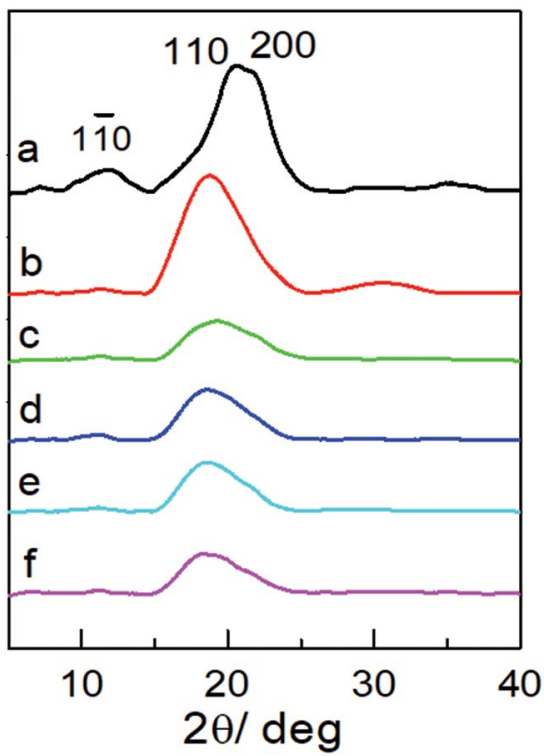

Fig. 3 XRD patterns of the NCG (a), PGMA (b) and the hydrophobic NCG-g-PGMA nanocomposites of S1 (c), S2 (d), S3 (e) and S4 (f).
NCG and PGMA, indicating that nothing interfered with the formation of the nanocomposite structure by SI-ATRP and hydrophobic modification. ${ }^{41,42,53}$

The microscopic morphologies and structures of the NCG, NCG- $g$-PGMA nanocomposite and the hydrophobic NCG- $g$ PGMA nanocomposites (Table 1, samples S1-S4) were examined by SEM (Fig. 4). The characteristic three-dimensional (3D) network of the cellulose nanofibrils was clearly visible on the surface of the NCG, NCG- $g$-PGMA nanocomposite and hydrophobic NCG-g-PGMA nanocomposites. Obviously, the NCG showed a homogeneous nanoporous structure consisting of pores approximately 50 to $150 \mathrm{~nm}$ in diameter and interconnected cellulose nanofibrils with a typical diameter of approximately 10 to $20 \mathrm{~nm}$ (Fig. $4 \mathrm{a}$ and b). After SI-ATRP of GMA monomers, the SEM images of the NCG- $g$-PGMA nanocomposite showed features consistent with grafting of PGMA (Fig. 4c and d). The NCG- $g$-PGMA nanocomposite consisted of rough nanofibrils with diameters of approximately 50 to $100 \mathrm{~nm}$ and large nanopores with diameters of 100 to $200 \mathrm{~nm}$, which were obviously associated with chemical grafting by PGMA. This result is also consistent with theoretical evidence suggesting that the mean pore size increases with an increase in the fiber diameter. ${ }^{54}$ Further hydrophobic modification with $\mathrm{C}_{7} \mathrm{~F}_{15} \mathrm{COCl}$ did not change the microscopic morphology and structure of the NCG- $g$-PGMA nanocomposite (Fig. 4i and l, Table 1, sample S4). However, the hydrophobic NCG-g-PGMA nanocomposite (sample S4) prepared at $30{ }^{\circ} \mathrm{C}$ exhibited a smoother surface, higher density $\left(1.3 \mathrm{~g} \mathrm{~cm}^{-3}\right)$, and smaller nanopores with diameters of 20 to $100 \mathrm{~nm}$ than the NCG- $g$-PGMA nanocomposite. This change in the microstructure resulted from the smoothing effect that the grafted polymers had on the surface, making the microstructure less rough. ${ }^{17}$ A very similar phenomenon was observed in the SEM images of the hydrophobic NCG- $g$-PGMA nanocomposites (Table 1, samples S3, S5S8) prepared with different grafting times (see the ESI, Fig. S1 $\dagger$ ). Therefore, the grafting temperature and time played an important role in the grafting of PGMA on the NCG and hence dramatically affected the microscopic morphology and structure of the hydrophobic NCG-g-PGMA nanocomposites.

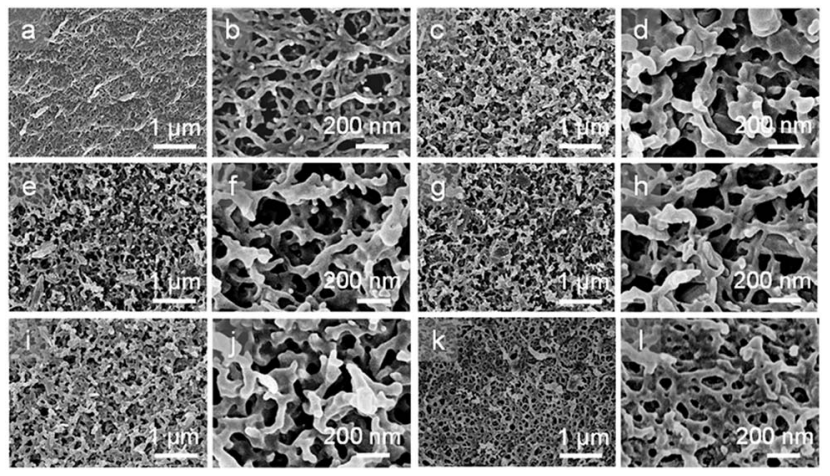

Fig. 4 SEM images of the surface of the NCG ( $a$ and b), the NCG-gPGMA nanocomposite (c and d) and the hydrophobic NCG-g-PGMA nanocomposites of S1 (e and f), S2 ( $g$ and h), S3 ( $i$ and j) and S4 ( $k$ and l) at low- and high-magnification. 
The hydrophobic behavior of the hydrophobic NCG- $g$-PGMA nanocomposites was further investigated by measuring the water contact angles (Fig. 5), which was strongly affected by the grafting temperature and time. The hydrophobicity of the NCG- $g$-PGMA nanocomposite increased considerably following SI-ATRP grafting of PGMA and hydrophobic modification with $\mathrm{C}_{7} \mathrm{~F}_{15} \mathrm{OCl}$. Water droplets stained with toluidine blue could be absorbed on the NCG aerogel freeze dried from water because of the capillary effect of the nanopores and the hydrophilicity of cellulose (Fig. 5a and c), and droplets exist and maintain a droplet shape on the surface of the hydrophobic NCG- $g$-PGMA nanocomposites (Table 1, sample S3) without being absorbed for a long time, which provides clear evidence of the high hydrophobicity of these materials (Fig. 5b and d). The polymerization of PGMA on the NCG to form the NCG- $g$ PGMA nanocomposite resulted in an increase in the water contact angle to $67^{\circ}$, indicating a significant improvement in the hydrophobicity. Expectedly, the low surface energy compound $\left(\mathrm{C}_{7} \mathrm{~F}_{15} \mathrm{OCl}\right)$ improved the hydrophobicity of the NCG- $g$-PGMA nanocomposite (Fig. 5e; Table 1, sample S3) because the water contact angle of air is considered to be $136^{\circ}$. The water contact angles of the hydrophobic NCG-g-PGMA nanocomposites gradually increased as the grafting temperature increased from 0 to $30{ }^{\circ} \mathrm{C}$ (Table 1 , samples S1-S4), and a maximum value of $136^{\circ}$ was achieved at a grafting temperature of $20^{\circ} \mathrm{C}$. At a grafting temperature of $30{ }^{\circ} \mathrm{C}$, the water contact angle of the hydrophobic NCG- $g$ PGMA nanocomposite decreased slightly to $121^{\circ}$, which was most likely due to the reduced micro- and nano-pore size of this hydrophobic NCG- $g$-PGMA nanocomposite, as shown in Fig. 4k and 1. Additionally, a very similar trend was observed for the hydrophobic NCG-g-PGMA nanocomposites when the grafting time was increased from 5 to $48 \mathrm{~h}$ (Table 1, samples S3, S5-S8). The hydrophobic NCG- $g$-PGMA nanocomposites exhibited a maximum
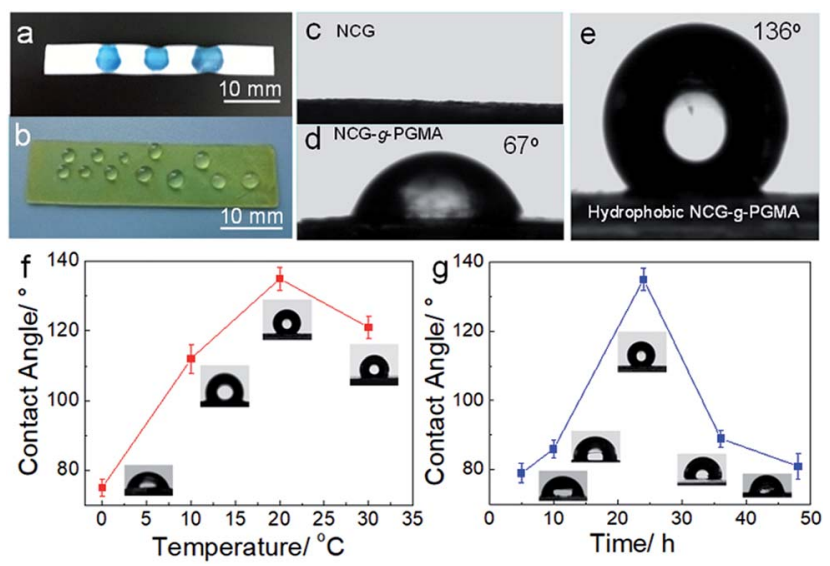

Fig. 5 (a and b) Photographs of a NCG aerogel (a) and a hydrophobic NCG-g-PGMA nanocomposite (b) with water droplets. (c-e) Photographs of the water contact angles on the surfaces of the NCG (c), NCG-g-PGMA nanocomposite (d) and hydrophobic NCG-g-PGMA nanocomposite (Table 1, sample S3) (e). Water contact angles of the hydrophobic NCG-g-PGMA nanocomposites prepared at different temperatures for $24 \mathrm{~h}$ (Table 1, samples S1-S4) (f) and with different reaction times at $20^{\circ} \mathrm{C}$ (Table 1 , samples S3, S5-S8) (g). The insets are the corresponding water contact angle photographs of the hydrophobic NCG-g-PGMA nanocomposites. All error bars represent the s.d. of at least three replicate measurements. water contact angle of $136^{\circ}$ at a grafting time of $24 \mathrm{~h}$, and then the contact angle decreased dramatically to $81^{\circ}$ at $48 \mathrm{~h}$. Compared with the hydrophobic NCG- $g$-PGMA nanocomposites, the water contact angle of $\mathrm{NCG}_{-} \mathrm{C}_{7} \mathrm{~F}_{15}$ was $84^{\circ}$ (not shown). The probable reason was that the amount of reactive hydroxyl groups of NCG was insufficient when using the same amount of hydrolyzed NCG$g$-PGMA nanocomposite in $\mathrm{C}_{7} \mathrm{~F}_{15} \mathrm{OCl}$, and the hydrophobic compound did not cover the entire the surface of NCG. Nevertheless, the water contact angles of the hydrophobic NCG- $g$-PGMA nanocomposites were dramatically increased via the grafting of PGMA but were not directly affected by the PGMA content, confirming again that the water contact angles of the hydrophobic NCG- $g$-PGMA nanocomposites were affected by the chemical composition of the surface and the micro- and nano-pore size. ${ }^{17}$

The DSC thermograms of the neat PGMA and the hydrophobic NCG-g-PGMA nanocomposites (Table 1, samples S1-S4) are shown in Fig. 6. The $T_{\mathrm{g}}$ of the neat PGMA in the nanocomposites was established by DSC, and the results are also summarized in Table 1. While neat PGMA showed a typical $T_{\mathrm{g}}$ value of approximately $68{ }^{\circ} \mathrm{C}$, the values shifted to higher temperatures in the presence of the NCG in the hydrophobic NCG- $g$-PGMA nanocomposites. For instance, the $T_{\mathrm{g}}$ of sample S1 was $85{ }^{\circ} \mathrm{C}$, and this value gradually decreased to $72{ }^{\circ} \mathrm{C}$ with increasing PGMA content in sample S4 (63 wt\% vs. $77 \mathrm{wt} \%$ ). Additionally, a very similar trend was observed as the PGMA content increased from $63 \mathrm{wt} \%$ to $80 \mathrm{wt} \%$ in the hydrophobic NCG- $g$-PGMA nanocomposites (Table 1, samples S3, S5-S8), in which the $T_{\mathrm{g}}$ value decreased from 84 to $65{ }^{\circ} \mathrm{C}$ (Table 1$)$. Here, the restriction of mobility was weakened as the proportion of PGMA in the nanocomposite increased, thus resulting in a decrease in the $T_{\mathrm{g}}{ }^{22}$ This change is also ascribed to the grafting of PGMA chains on cellulose nanofibrils by SI-ATRP and the confinement effect of the interconnected cellulose nanofibril networks of the NCG. ${ }^{\mathbf{4 1 , 4 2}}$

Fig. 7 shows the dynamic mechanical behavior of the NCG, PGMA and hydrophobic NCG- $g$-PGMA nanocomposites (Table 1, samples S1-S4) determined by DMA at $1 \mathrm{~Hz}$. Neat PGMA showed

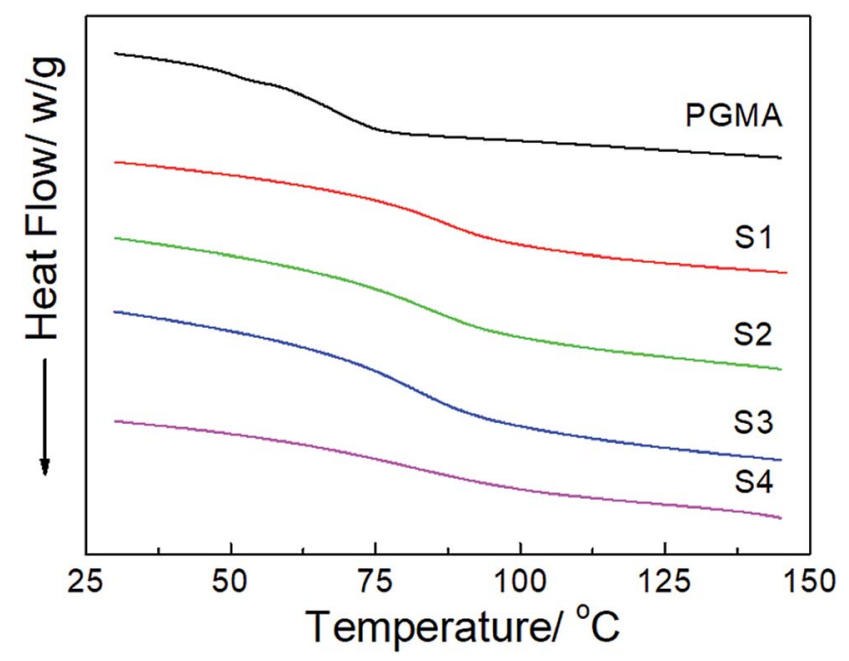

Fig. 6 DSC thermograms of neat PGMA and the hydrophobic NCG-gPGMA nanocomposites (Table 1, samples S1-S4). 
behavior typical of an amorphous polymer. Below $60{ }^{\circ} \mathrm{C}$, neat PGMA was in the glassy state and exhibited a slight decrease in the tensile storage modulus $\left(E^{\prime}\right)$ from 35 to $60{ }^{\circ} \mathrm{C}$ because of the restriction of molecular motion. A sudden drop in $E^{\prime}$ occurred above $60{ }^{\circ} \mathrm{C}$, which resulted from the motion of the PMGA chain segments. In comparison, most of the hydrophobic NCG- $g$-PGMA nanocomposites exhibited a modest increase in $E^{\prime}$ below the $T_{\mathrm{g}}$ of PGMA. Remarkably, the incorporation grafting time of $24 \mathrm{~h}$, and then the contact angle decreased dramatically to $81^{\circ}$ at $48 \mathrm{~h}$. Therefore, the contact angles of the hydrophobic NCG- $g$-PGMA nanocomposites were dramatically increased via the grafting of PGMA but were not directly affected by of the NCG drastically changed the mechanical behavior of the hydrophobic NCG- $g$ PGMA nanocomposites above their $T_{\mathrm{g}}$, where they displayed significant mechanical reinforcement behavior as a result of the grafting from the NCG. For example, at $180{ }^{\circ} \mathrm{C}$, the $E^{\prime}$ of hydrophobic NCG- $g$-PGMA nanocomposite (Table 1 , sample S1) was 337-fold higher than that of PGMA (117.9 MPa vs. 0.35 MPA). In the rubbery state, the $E^{\prime}$ of hydrophobic NCG- $g$-PGMA nanocomposite S4 with $23 \mathrm{wt} \%$ NCG was lower than that of sample S1 with 37 wt $\%$ NCG. This difference could be attributed to stronger
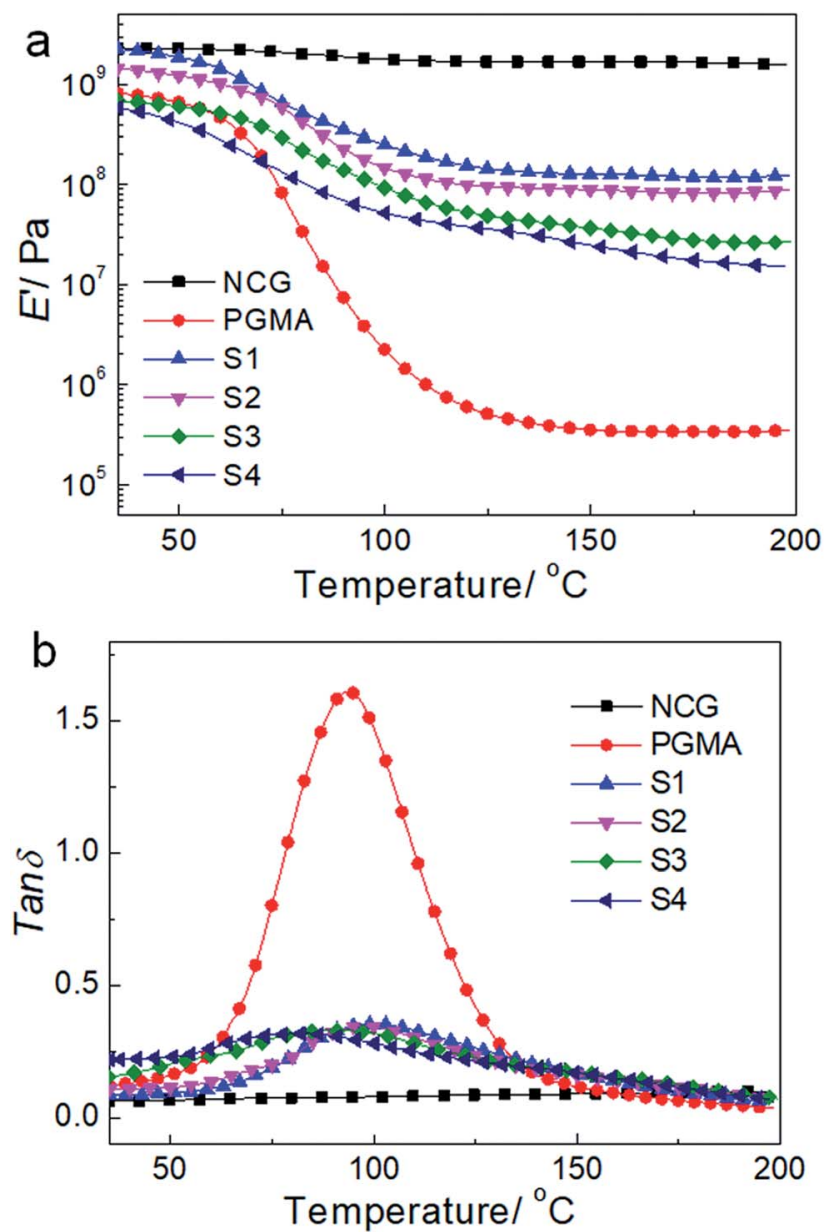

Fig. 7 DMA temperature sweeps for the NCG, PGMA and the hydrophobic NCG-g-PGMA nanocomposites. Storage modulus $E^{\prime}(a)$ and loss tangents $\tan \delta$ (b) of the NCG, PGMA and nanocomposites as a function of temperature.
NCG-polymer adhesion in the interfacial zone due to the presence of additional interactions. Similar reinforcement effects of polymers above the $T_{\mathrm{g}}$ have also been reported in bacterial cellulose- $g$-poly (methyl methacrylate) nanocomposites ${ }^{53}$ and cotton fabric- $g$-poly(tert-butyl acrylate). ${ }^{55}$ Notably, the PGMA grafted in the hydrophobic NCG- $g$-PGMA nanocomposites had a significantly lower $M_{\mathrm{n}}$ (less than $9.4 \times 10^{3} \mathrm{~g} \mathrm{~mol}^{-1}$ ) than neat PGMA $\left(1.3 \times 10^{4} \mathrm{~g} \mathrm{~mol}^{-1}\right)$. Moreover, the molecular weight of the grafted PGMA appeared to have no direct effect on the mechanical properties of the hydrophobic NCG- $g$-PGMA nanocomposites. Thus, the significant reinforcement effect of the hydrophobic NCG- $g$-PGMA nanocomposites is attributable to the strong mobility was weakened as the proportion of PGMA in the intermolecular hydrogen bonding interactions and the confinement effects of the entangled cellulose chains within the interconnected nanofibrillar network structure of the NCG. ${ }^{\mathbf{4 1 , 4 2}}$ Moreover, the maximum loss tangent $(\tan \delta)$ exhibited a higher $\alpha$ relaxation temperature in the hydrophobic NCG- $g$-PGMA nanocomposites ( $T_{\alpha}$ of approximately $100{ }^{\circ} \mathrm{C}$ ) than in neat PGMA (approximately $94{ }^{\circ} \mathrm{C}$ ) (Fig. 7b). Additionally, the intensity of the $\tan \delta$ peak decreased for the hydrophobic NCG- $g$-PGMA
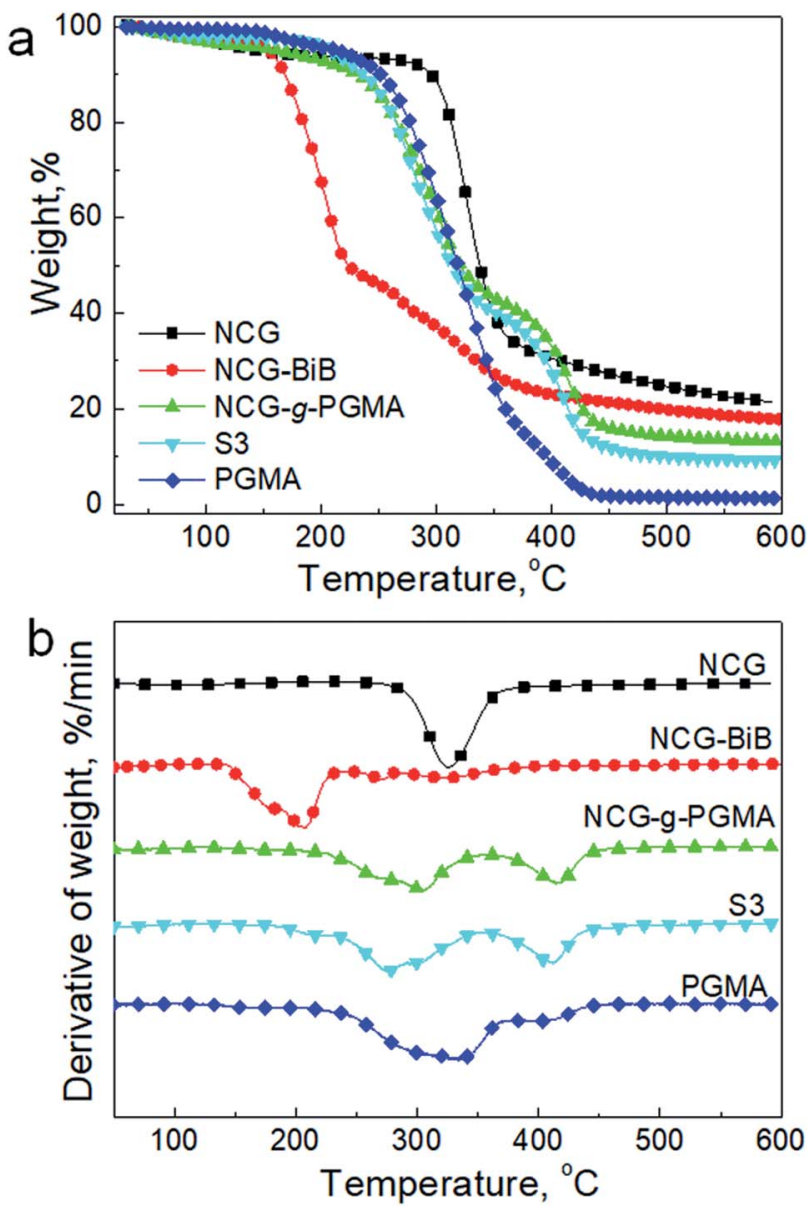

Fig. 8 TGA results (a) and derivative thermogravimetry (DTG) curves (b) of the NCG, NCG-BiB, NCG-g-PGMA nanocomposite, hydrophobic NCG-g-PGMA nanocomposite (Table 1, sample S3) and PGMA under a nitrogen atmosphere. 
nanocomposites, confirming the grafting of PGMA on cellulose nanofibrils of the NCG and the confinement effect of the cellulose nanofibril networks of the NCG, which substantially reduced the chain relaxation of PGMA. ${ }^{33,41,42}$

We further examined the thermal decomposition of the NCG, NCG-BiB, NCG-g-PGMA nanocomposite and a hydrophobic NCG- $g$-PGMA nanocomposite (sample S3) by TGA under nitrogen, as shown in Fig. 8. Although thermal decomposition of the cellulose nanowhiskers occurred below $150{ }^{\circ} \mathrm{C}$ because of the presence of sulfate groups, the NCG withstood heating up to $290-370{ }^{\circ} \mathrm{C}$, which is the decomposition temperature of pristine cellulose.$^{\mathbf{4 1 , 4 2}}$ The introduction of $\mathrm{BiB}$ groups resulted in a decrease in the thermal stability of the NCG, with the decomposition peak observed at $205^{\circ} \mathrm{C}$. The decrease in thermal stability for NCG-BiB was attributed to the degradation of the bromoalkyl groups to release $\mathrm{HBr}$ upon heating, which induces cellulose degradation at lower temperatures. ${ }^{29,53}$ In contrast, the thermograms of the NCG- $g$ PGMA nanocomposite and hydrophobic NCG- $g$-PGMA nanocomposite (Table 1, sample S3) showed a substantial increase in stability relative to the corresponding NCG-BiB. The NCG- $g$ PGMA nanocomposite exhibited two decomposition peaks at approximately $305{ }^{\circ} \mathrm{C}$ and $415{ }^{\circ} \mathrm{C}$, which can be attributed to the thermal decomposition of the NCG and PGMA components of the NCG- $g$-PGMA nanocomposite. ${ }^{56}$ After functionalization with $\mathrm{C}_{7} \mathrm{~F}_{15} \mathrm{COCl}$, the decomposition peaks of NCG and PGMA of hydrophobic NCG- $g$-PGMA nanocomposite slightly decreased to $276{ }^{\circ} \mathrm{C}$ and $411^{\circ} \mathrm{C}$. Since the hydrophobic NCG- $g$ PGMA nanocomposites did not melt or decompose up to $250{ }^{\circ} \mathrm{C}$, they can be used at much higher temperatures than the neat polymers.

\section{Conclusions}

In summary, the hydrophobic NCG- $g$-PGMA nanocomposites were fabricated via SI-ATRP of GMA monomers and hydrophobic modification with $\mathrm{C}_{7} \mathrm{~F}_{15} \mathrm{COCl}$ on the cellulose nanofibrils of the NCG. The grafting temperature and time played an important role in grafting PGMA on NCG and hence dramatically affected the microscopic morphology and structure of the hydrophobic NCG-g-PGMA nanocomposites. The interconnected nanofibrillar network structure of the NCG was deposited with PGMA after SI-ATRP. Further hydrophobic modification with $\mathrm{C}_{7} \mathrm{~F}_{15} \mathrm{COCl}$ did not change the microscopic morphology and structure of the NCG- $g$-PGMA nanocomposites. Moreover, the hydrophobic NCG-g-PGMA nanocomposites demonstrated a remarkable improvement in the tensile storage modulus above the glass transition temperature. The presented results illustrated the usefulness of NCG as platform for SI-ATRP and provided potential opportunities for fabricating functional NCG-based nanomaterials.

\section{Conflicts of interest}

There are no conflicts to declare.

\section{Acknowledgements}

This research was funded by the National Natural Science Foundation of China $(21422405,51373125)$ and the Major Program of National Natural Science Foundation of China (21334005). The authors thank to the facility support of the Wuhan Morning Light Plan of Youth Science and Technology (2017050304010312), the Special Fund for the Development of Strategic Emerging Industries of Shenzhen City of China (JCYJ20170818112409808), and Fundamental Research Funds for the Central Universities (2042017kf0195, 2042015kf0259, 2042017kf0227).

\section{References}

1 S. J. Eichhorn, A. Dufresne, M. Aranguren, N. E. Marcovich, J. R. Capadona, S. J. Rowan, C. Weder, W. Thielemans, M. Roman, S. Renneckar, W. Gindl, S. Veigel, J. Keckes, H. Yano, K. Abe, M. Nogi, A. N. Nakagaito, A. Mangalam, J. Simonsen, A. S. Benight, A. Bismarck, L. A. Berglund and T. Peijs, J. Mater. Sci., 2010, 45, 1-33.

2 D. Klemm, F. Kramer, S. Moritz, T. Lindstrom, M. Ankerfors, D. Gray and A. Dorris, Angew. Chem., Int. Ed., 2011, 50, 54385466.

3 Y. Habibi, Chem. Soc. Rev., 2014, 43, 1519-1542.

4 J. G. Torres-Rendon, T. Femmer, L. De Laporte, T. Tigges, K. Rahimi, F. Gremse, S. Zafarnia, W. Lederle, S. Ifuku, M. Wessling, J. G. Hardy and A. Walther, Adv. Mater., 2015, 27, 2989-2995.

5 H. Zhu, W. Luo, P. N. Ciesielski, Z. Fang, J. Y. Zhu, G. Henriksson, M. E. Himmel and L. Hu, Chem. Rev., 2016, 116, 9305-9374.

6 R. Weishaupt, G. Siqueira, M. Schubert, M. M. Kämpf, T. Zimmermann, K. Maniura-Weber and G. Faccio, Adv. Funct. Mater., 2017, 27, 1604291.

7 S. Wang, T. Li, C. Chen, W. Kong, S. Zhu, J. Dai, A. J. Diaz, E. Hitz, S. D. Solares, T. Li and L. Hu, Adv. Funct. Mater., 2018, 28, 1707491.

8 M. Zhao, F. Ansari, M. Takeuchi, M. Shimizu, T. Saito, L. A. Berglund and A. Isogai, Nanoscale Horiz., 2018, 3, 28-34.

9 A. Sydney Gladman, E. A. Matsumoto, R. G. Nuzzo, L. Mahadevan and J. A. Lewis, Nat. Mater., 2016, 15, 413.

10 J. O. Zoppe, N. C. Ataman, P. Mocny, J. Wang, J. Moraes and H. Klok, Chem. Rev., 2017, 117, 1105-1318.

11 J. Ran, L. Wu, Z. Zhang and T. Xu, Prog. Polym. Sci., 2014, 39, 124-144.

12 O. Garcia Valdez, P. Champagne and M. F. Cunningham, Prog. Polym. Sci., 2018, 76, 151-173.

13 V. S. Vo, S. Mahouche Chergui, J. Babinot, V. H. Nguyen, S. Naili and B. Carbonnier, RSC Adv., 2016, 6, 89322-89327.

14 A. Khabibullin, K. Bhangaonkar, C. Mahoney, Z. Lu, M. Schmitt, A. K. Sekizkardes, M. R. Bockstaller and K. Matyjaszewski, ACS Appl. Mater. Interfaces, 2016, 8, 5458-5465.

15 K. Matyjaszewski, Adv. Mater., 2018, 30, 1706441.

16 D. Roy, M. Semsarilar, J. T. Guthrie and S. Perrier, Chem. Soc. Rev., 2009, 38, 2046-2064. 
17 S. Hansson, E. Ostmark, A. Carlmark and E. Malmstrom, ACS Appl. Mater. Interfaces, 2009, 1, 2651-2659.

18 D. Nyström, J. Lindqvist, E. Östmark, P. Antoni, A. Carlmark, A. Hult and E. Malmström, ACS Appl. Mater. Interfaces, 2009, 1, 816-823.

19 H. Wu, L. Wu, S. Lu, X. Lin, H. Xiao, X. Ouyang, S. Cao, L. Chen and L. Huang, Carbohydr. Polym., 2018, 181, 419425.

20 L. Zhao, L. Li, Y. Wang, J. Wu, G. Meng, Z. Liu and X. Guo, Appl. Phys. A: Mater. Sci. Process., 2018, 124, 9.

21 J. Lindqvist, D. Nystrom, E. Ostmark, P. Antoni, A. Carlmark, M. Johansson, A. Hult and E. Malmstrom, Biomacromolecules, 2008, 9, 2139-2145.

22 J. O. Zoppe, Y. Habibi, O. J. Rojas, R. A. Venditti, L. Johansson, K. Efimenko, M. Österberg and J. Laine, Biomacromolecules, 2010, 11, 2683-2691.

23 R. Alosmanov, K. Wolski and S. Zapotoczny, Cellulose, 2017, 24, 285-293.

24 J. Arredondo, P. G. Jessop, P. Champagne, J. Bouchard and M. F. Cunningham, Green Chem., 2017, 19, 4141-4152.

25 K. Ou, X. Wu, B. Wang, C. Meng, X. Dong and J. He, Cellulose, 2017, 24, 5211-5224.

26 A. Aied, Y. Zheng, A. Pandit and W. Wang, ACS Appl. Mater. Interfaces, 2012, 4, 826-831.

27 B. V. Bhut, K. A. Conrad and S. M. Husson, J. Membr. Sci., 2012, 390-391, 43-47.

28 A. Boujemaoui, C. C. Sanchez, J. Engstrom, C. Bruce, L. Fogelstrom, A. Carlmark and E. Malmstrom, ACS Appl. Mater. Interfaces, 2017, 9, 35305-35318.

29 Z. Zhang, G. Sèbe, X. Wang and K. C. Tam, ACS Appl. Nano Mater., 2018, 1, 632-641.

30 M. Morits, J. R. McKee, J. Majoinen, J. M. Malho, N. Houbenov, J. Seitsonen, J. Laine, A. H. Gröschel and O. Ikkala, ACS Sustainable Chem. Eng., 2017, 5, 7642-7650.

31 J. R. McKee, J. Huokuna, L. Martikainen, M. Karesoja, A. Nykänen, E. Kontturi, H. Tenhu, J. Ruokolainen and O. Ikkala, Angew. Chem., Int. Ed., 2014, 53, 5049-5053.

32 J. Cai, S. Kimura, M. Wada, S. Kuga and L. Zhang, Chemsuschem, 2008, 1, 149-154.

33 Z. Shi, J. Huang, C. Liu, B. Ding, S. Kuga, J. Cai and L. Zhang, ACS Appl. Mater. Interfaces, 2015, 7, 22990-22998.

34 D. Zhao, J. Huang, Y. Zhong, K. Li, L. Zhang and J. Cai, Adv. Funct. Mater., 2016, 26, 6279-6287.

35 J. Cai, S. Liu, J. Feng, S. Kimura, M. Wada, S. Kuga and L. Zhang, Angew. Chem., Int. Ed., 2012, 51, 2076-2079.
36 J. Cai, S. Kimura, M. Wada and S. Kuga, Biomacromolecules, 2009, 10, 87-94.

37 Q. Wang, J. Cai and L. Zhang, Cellulose, 2014, 21, 3371-3382.

38 D. Xu, C. Chen, J. Xie, B. Zhang, L. Miao, J. Cai, Y. Huang and

L. Zhang, Adv. Energy Mater., 2016, 6, 1501929.

39 Z. Shi, H. Gao, J. Feng, B. Ding, X. Cao, S. Kuga, Y. Wang, L. Zhang and J. Cai, Angew. Chem., Int. Ed., 2014, 53, 53805384.

40 Q. Wang, J. Cai, L. Zhang, M. Xu, H. Cheng, C. C. Han, S. Kuga, J. Xiao and R. Xiao, J. Mater. Chem. A, 2013, 1, 6678-6686.

41 K. Li, J. Huang, H. Gao, Y. Zhong, X. Cao, Y. Chen, L. Zhang and J. Cai, Biomacromolecules, 2016, 17, 1506-1516.

42 K. Li, J. Song, M. Xu, S. Kuga, L. Zhang and J. Cai, ACS Appl. Mater. Interfaces, 2014, 6, 7204-7213.

43 J. Cai and L. Zhang, Macromol. Biosci., 2005, 5, 539-548.

44 A. Carlmark and E. Malmstrom, J. Am. Chem. Soc., 2002, 124, 900-901.

45 A. Carlmark and E. E. Malmstrom, Biomacromolecules, 2003, 4, 1740-1745.

46 D. Daniel, X. Chunlin, L. Otto, H. Jarl, B. Peter and W. Stefan, J. Polym. Sci., Part A: Polym. Chem., 2013, 51, 5100-5110.

47 D. J. Siegwart, J. K. Oh and K. Matyjaszewski, Prog. Polym. Sci., 2012, 37, 18-37.

48 Y. Maréchal and H. Chanzy, J. Mol. Struct., 2000, 523, 183196.

$49 \mathrm{~S} . \mathrm{Li}, \mathrm{M}$. Xiao, A. Zheng and H. Xiao, Biomacromolecules, 2011, 12, 3305-3312.

50 X. Sui, J. Yuan, M. Zhou, J. Zhang, H. Yang, W. Yuan, Y. Wei and C. Pan, Biomacromolecules, 2008, 9, 2615-2620.

51 Z. Zhang, Q. Wu, K. Song, T. Lei and Y. Wu, Cellulose, 2015, 22, 2431-2441.

52 D. Nyström, J. Lindqvist, E. Ostmark, A. Hult and E. Malmström, Chem. Commun., 2006, 34, 3594.

53 P. S. S. Lacerda, A. M. M. V. Barros-Timmons, C. S. R. Freire, A. J. D. Silvestre and C. P. Neto, Biomacromolecules, 2013, 14, 2063-2073.

54 S. J. Eichhorn and W. W. Sampson, J. R. Soc., Interface, 2005, 2, 309-318.

55 X. Dong, H. Bao, K. Ou, J. Yao, W. Zhang and J. He, Fibers Polym., 2015, 16, 1478-1486.

56 N. Misra, N. K. Goel, S. A. Shelkar, L. Varshney and V. Kumar, J. Mol. Catal. B: Enzym., 2016, 133, S172-S178. 